\title{
Effect of surface nitridation on the epitaxial growth of few-layer $\mathrm{sp}^{2} \mathrm{BN}$
}

\author{
Michael Snure *, Qing Paduano, and Arnold Kiefer
}

Air Force Research Laboratory, Sensors Directorate, Wright-Patterson AFB, OH 45433, USA

Boron Nitride is a promising 2D dielectric material for use in numerous electronic applications.

In order to realize this potential, a process for producing atomically thin layers on

microelectronics-compatible substrates is desirable. In this paper we describe an approach to

epitaxially grow few-layer $\mathrm{sp}^{2} \mathrm{BN}$ directly on an insulating substrate, using metal-organic

chemical vapor deposition (MOCVD). We also elucidate the effect of sapphire surface

nitridation on the growth characteristics. We compare the effect of nitridation on the growth rate, surface morphology and structure across a wide range of V/III ratios. Depending on the V/III ratio, two different growth modes were identified: at low V/III 3D island growth is dominant and at high V/III the growth transitions to a self-terminating mode. Under self-terminating growth a film thickness of $1.5 \mathrm{~nm}$ is typically achieved. Surface nitridation was found to improve nucleation, promoting self-terminating growth, and resulting in atomically smooth films. Reflection high energy electron diffraction (RHEED) patterns reveal the epitaxial relationship between $\mathrm{BN}$ and sapphire to be [1-100]\|[11-20] and [0001]\|[0001]. Growth at low V/III ratios without surface nitridation produced films with large hexagonal holes, which could not be completely filled by extending the growth time. Through surface nitridation, these holes were eliminated, producing continuous smooth films.

Keywords: A3.Metal Organic Chemical Vapor Deposition, B1.Nitrides, B2.Dielectric Materials;

\section{A1.Surface Structure}

*Author to whom correspondence should be addressed; E-mail: michael.snure.1@us.af.mil, Tel: $+1-973-528-8929$ 


\section{Introduction}

Boron nitride, with $\mathrm{sp}^{2}$ bonding, is a layered material structurally similar to graphite with strong in-plane bonding and very weak out-of-plane van der Waals bonding. Like graphene, individual layers of $\mathrm{sp}^{2} \mathrm{BN}$ are atomically thin and smooth and can be easily isolated. The basal plane is constructed of $\mathrm{sp}^{2}$ bonded $\mathrm{B}$ to $\mathrm{N}$ forming a hexagonal lattice. In few to bulk BN, layers are known to stack in three sequences $\mathrm{AB}$ (hexagonal $\mathrm{BN}), \mathrm{ABC}$ (rhombohedral $\mathrm{BN}$ ) and random (turbostratic BN) with only very small differences in interplanar spacing [1]. Unlike graphene, the $\mathrm{sp}^{2} \mathrm{~B}-\mathrm{N}$ bonds are partially ionic, making $\mathrm{BN}$ a wide band gap insulator [2]. These properties make $\mathrm{sp}^{2} \mathrm{BN}$ a promising two dimensional (2D) dielectric material, well suited as a substrate and gate dielectric for 2D electronics. The most widely used approaches for producing mono to few-layer BN have been mechanical exfoliation from bulk sources or chemical vapor deposition (CVD) on catalyzing metal substrates. Exfoliation is a very simple approach but is not currently suitable for large area device fabrication. While CVD BN can be grown over large areas on metal substrates like $\mathrm{Cu}$ foil, transfer to a host substrate is still required for device fabrication. Ideally, heterostructures of 2D materials would be grown directly on microfabrication compatible substrates.

Initial work on CVD growth of BN indicated that temperatures in excess of $1500{ }^{\circ} \mathrm{C}$ would be required to epitaxially grow h-BN [3]. In more recent work epitaxial growth of h-BN on sapphire was demonstrated by MOCVD at $1080^{\circ} \mathrm{C}$ [4], while at $1500{ }^{\circ} \mathrm{C}$ epitaxial growth of the rhombohedral phase of BN on sapphire was demonstrated [5]. These studies focused mostly on producing thick films. For 2D device applications, controlled growth of few-layer BN films needs to be achieved. Development of a self-terminating CVD/MOCVD process on insulating substrates similar to what has been done on single-crystal transition metals in UHV [6,7] is 
desirable for producing few- to mono-layer films with uniform thickness over large areas. In our previous report on growth of $\mathrm{BN}$ by MOCVD [8], we showed that atomically smooth few-layer thick BN can be achieved under a self-terminating growth mode at high V/III ratios.

Since growth behavior is sensitive to substrate surface properties, modification of the substrate surface is expected to play an important role in the growth of BN films. For instance, Chubarov et al. [9] reported that an AlN buffer layer is required for epitaxial growth of BN. Another way to modify the surface is nitridation. Early sapphire nitridation studies in MOCVD observed the formation of an amorphous aluminum oxynitride $\left(\mathrm{AlN}_{\mathrm{x}} \mathrm{O}_{\mathrm{y}}\right)$ layer $[10,11]$, while other studies presented evidence for the formation of relaxed crystalline AlN on the sapphire surface $[12,13]$. The surface morphology of sapphire after nitridation was dependent on nitridation conditions [14], such as nitridation time and temperature, etc.

From our previous work [8] we realize that under the temperatures and V/III ratios required to grow atomically smooth $\mathrm{BN}$, unintentional nitridation is likely to occur. Therefore, in this work we study the effects of sapphire surface nitridation on the growth of ultra-thin $\mathrm{sp}^{2}$ $\mathrm{BN}$ films within a MOCVD system. Our nitridation process is performed at a temperature of $900^{\circ} \mathrm{C}$, with a low ammonia-to-hydrogen ratio, for 10 minutes. Sapphire substrates treated with this nitridation process were verified to have a layer of $\operatorname{AlN}_{\mathrm{x}} \mathrm{O}_{\mathrm{y}}$ on the surface. We show herein that nitridation has a significant effect on the nucleation of 2D BN on sapphire, impacting the BN thickness and surface morphology as characterized by atomic force microscopy (AFM), Xray reflectance (XRR), reflection high energy electron diffraction (RHEED) and Raman spectroscopy.

\section{Experiment}


Boron nitride growth experiments were performed in a low pressure MOCVD reactor with an actively cooled, close-coupled showerhead. A growth temperature of $1100{ }^{\circ} \mathrm{C}$ and a reactor pressure of 20 Torr were used to achieve self-terminating growth [8]. Triethylboron (TEB), $\mathrm{NH}_{3}$ and $\mathrm{H}_{2}$ were used as the boron precursor, nitrogen precursor and carrier gas, respectively. The V/III ratio was varied between 450 and 2700 by fixing the TEB molar flux at $10 \mu \mathrm{mol} / \mathrm{min}$ and changing the $\mathrm{NH}_{3}$ flow rate from 100 to $600 \mathrm{sccm}$. Epi-ready c-plane sapphire substrates with a $0.3^{\circ}$ miscut towards the m-plane were used. Nitridation of these substrates was performed by annealing at $900^{\circ} \mathrm{C}$ at a pressure of 300 Torr with $1 \% \mathrm{NH}_{3}$ in $\mathrm{H}_{2}$ for $10 \mathrm{~min}$. The pressure, temperature and flow rates were then adjusted to the $\mathrm{BN}$ growth conditions after the completion of nitridation.

The BN film thickness was determined from XRR measurements using a PANalytical Empyrean x-ray diffractometer in a grazing-incidence configuration. The film thickness was obtained by fitting to the measured reflectance using a three-layer model with the BN density constrained between 2 and $2.9 \mathrm{~g} / \mathrm{cm}^{3}$, and a layer of $\mathrm{AlO}_{\mathrm{x}} \mathrm{N}_{\mathrm{y}}$ was included at the interface between $\mathrm{BN}$ film and sapphire substrate. For fitting, the $\mathrm{AlO}_{\mathrm{x}} \mathrm{N}_{\mathrm{y}}$ thickness and density were constrained between 0 and $5 \mathrm{~nm}$ and 3 to $4 \mathrm{~g} / \mathrm{cm}^{3}$ as established from XRR measurements on reference sapphire substrates after nitridation (a typical density of $3.4 \mathrm{~g} / \mathrm{cm}^{3}$ was measured). We noticed that the $\mathrm{AlO}_{\mathrm{x}} \mathrm{N}_{\mathrm{y}}$ is not stable under atmospheric conditions as reported in ref. [15]. This was observed as a change in XRR results measured on the nitrided sapphire over time. To avoid inconsistency we conduct XRR measurements for each film within 30 min after unloading the films from the reactor.

Raman spectroscopy was used to identify the bonding nature of BN using a Renishaw inVia system. An accumulation of 120 scans, each of 30-second duration, was collected using a 
$4 \mathrm{~mW} 488 \mathrm{~nm}$ excitation source, $20-\mu \mathrm{m}$ slits and 3000 line/mm grating for each measurement. The surface structure of these ultra-thin films was characterized using reflection high-energy electron diffraction (RHEED). For RHEED measurements, samples were transferred to a separate vacuum system and then a $20-\mathrm{keV}$ electron beam was used to examine the surface. Surface topography of the BN films was analyzed by atomic force microscopy (AFM) using a Bruker Dimension Icon in tapping mode. X-ray photoelectron spectroscopy (XPS) was used to characterize the chemical composition and bonding environment of films using a PHI (PerkinElmer) 55000 XPS with an $\mathrm{Al} \mathrm{K}_{\alpha}$ X-ray source.

\section{Results}

The effect of nitridation time was studied for annealing times between $15 \mathrm{sec}$ and $30 \mathrm{~min}$, in order to determine the time required to controllably produce a smooth nitrided surface for growth of few-layer BN. After nitridation, the sapphire surface was characterized using XPS, XRR and AFM to determine chemical composition, thickness and morphology (Fig. 1). XPS showed the presence of the N1s peak indicating the incorporation of $\mathrm{N}$ into the sapphire surface for all nitridation times. Figure 1a shows the N1s XPS spectra from the sapphire surface after 1 min and 10 min of nitridation. We can see a change in the N1s peak shape as the nitridation time is increased. Deconvolution of the N1s peak into two components reveals peaks at 396.8 and 398. $1 \mathrm{eV}$, which can be attributed to the $\mathrm{N}-\mathrm{Al}$ and $\mathrm{N}-\mathrm{O}$ bonds [14-16]. Comparison of the $1 \mathrm{~min}$ and 10 min nitrided samples shows an increase in the $\mathrm{N}$-Al peak intensity with time, similar to what was reported by Diwikusuma and Kuech [16]. The presence of significant N-O bonding indicates the formation of amorphous $\mathrm{AlN}_{\mathrm{x}} \mathrm{O}_{\mathrm{y}}$ at the sapphire surface [15].

XRR curves from sapphire substrates nitrided for $15 \mathrm{sec}, 1 \mathrm{~min}, 10 \mathrm{~min}$ and $30 \mathrm{mins}$ are shown in Fig. 1b. From these curves we see the formation of a thin layer at the surface. The 
thickness of this layer increases with time, up to $10 \mathrm{~min}$, reaching a thickness of $\sim 1.5 \mathrm{~nm}$. For nitridation times longer than $10 \mathrm{~min}$, no increase in thickness was observed. Characterization of the surface morphology found that the roughness changed only slightly with time, increasing from 0.1 to $0.15 \mathrm{~nm}$ as the time was increased from $15 \mathrm{sec}$ to $30 \mathrm{~min}$. From the AFM images in Fig. 1c we can see that the step structure on the sapphire substrate is preserved even after nitridation. Based on these results a nitridation time of $10 \mathrm{~min}$ was adopted as the time that most reproducibly results in a smooth $\mathrm{AlN}_{\mathrm{x}} \mathrm{O}_{\mathrm{y}}$ surface layer ideal for growth of atomically smooth and thin $\mathrm{BN}$ growth.

Boron nitride samples grown at $1100{ }^{\circ} \mathrm{C}$ were produced both with and without the pregrowth nitridation process to study the effect of surface nitridation on morphology across a wide range of V/III ratios. Figure 2a shows AFM images from $\mathrm{BN}$ films grown for $15 \mathrm{~min}$ at $1100{ }^{\circ} \mathrm{C}$ for V/III ratios of 450, 900, 1350, and 2250 without nitridation. Under these growth conditions, film roughness decreases with increasing V/III ratio. An atomically smooth surface was achieved at high V/III (>1800). However, at moderate V/III ratios of 900 and 1350, a large number of holes of varying size and geometric shape were observed. Figure $2 b$ shows the surface morphology of BN films grown under the same growth conditions after the 10 min nitridation process. Compared to the AFM results shown in Fig. 2a, roughness decreases for the same V/III ratio with nitridation, indicating earlier onset of self-terminating growth (see Fig.2c); in concurrence with this conjecture, holes were completely eliminated on these films. Therefore, based on the interpretation of holes in films as incomplete coalescence, we believe that proper nitridation can improve nucleation for 2D film growth. Formation of holes in $\mathrm{BN}$ films without nitridation occurs in a narrow range of V/III ratios (900 to 1350). Below this V/III range, the growth is not self-terminating and 3D island growth results in a rough surface. At V/III ratios 
above 1800, these holes were not observed and the film is smooth (RMS $<0.2 \mathrm{~nm}$ ) and continuous, consistent with self-terminating growth. With nitridation, the transition to a smooth morphology with an RMS $<0.2 \mathrm{~nm}$ occurs at a V/III ratio of 1350 , and no holes are observed between 900 and 1350. It is likely that even the un-nitrided substrates experience unintentional nitridation at high $\mathrm{V} / \mathrm{III}$, thus achieving a similar result as performing an additional nitridation step prior to growth.

X-ray reflectance curves for $\mathrm{BN}$ films grown without the nitridation step across a wide range of V/III ratios are shown in Fig. 3a. Simulations were used to fit these curves and obtain the BN thicknesses (solid symbols in Fig. 3b); similar curves (not shown) were used to determine thicknesses with nitridation (open symbols in Fig. 3b). At a high V/III ratio (>1800) the BN thickness remains constant at $\sim 1.5 \mathrm{~nm}$, corresponding to an average thickness of 5 layers, which is consistent with our previous report for self-terminating growth [8]. The inset of Fig. 3b shows self-terminating growth at a V/III ratio of 2250 represented by a constant thickness with growth time. As the V/III ratio is reduced from 1800 to 900, a slight decrease in fringe spacing is observed in Fig. 3a, indicating a small increase in average film thickness. A further decrease in V/III ratio to 450 causes a significant change in thickness, corresponding to a shift in the growth mode to a 3D type growth. Films produced using the nitridation process have a similar trend of decrease in thickness with V/III ratio (average thickness is slightly lower). Surface nitridation appears to promote self-terminating growth, thus decreasing the V/III ratio required for this growth mode.

Raman was used to characterize the phase and bonding in these films. The characteristic Raman shift at $1369 \mathrm{~cm}^{-1}$, corresponding to the in-plane $\mathrm{E}_{2 \mathrm{~g}}$ phonon mode of $\mathrm{sp}^{2} \mathrm{BN}$, was used for identification [17,18]. Spectra from films grown with and without nitridation at low (450) 
and high V/III ratios (2250) are presented in Fig. 4a. An obvious decrease in the Raman intensity with increasing V/III ratio is observed, which is attributed to the reduction in thickness. Pre-growth nitridation leads to a substantial decrease in the Raman intensity at low V/III ratios while having no observable effect at high V/III ratios. Again, these differences are explained by changes in thickness, which at a low V/III ratio reduces by $25 \%$ with surface nitridation. The $\mathrm{E}_{2 \mathrm{~g}}$ peak position was found to be $\sim 1372 \mathrm{~cm}^{-1}$ for all four samples. However, the FWHM decreased from 30 to $25 \mathrm{~cm}^{-1}$ as the V/III was increased from 450 to 2250 indicating an improvement in crystallinity consistent with the observed change in film morphology [18]. A similar degree of broadening and shifting in the BN Raman peak was observed by Ismach et al.[19] and Tay et al. [20], who reported CVD growth of h-BN thin films with grain sizes of several microns.

XPS analysis of our BN films found the B/N to be very consistent across a wide range of V/III ratios, ranging from $1 / 1$ to $1 / 1.05$ from film to film. Figure $4 \mathrm{~b}$ shows XPS spectra from the $\mathrm{B} 1 \mathrm{~s}$ and N1s peaks of $\mathrm{BN}$ films grown at a V/III of 2250 with and without the nitridation process. Only one peak is observed in the B1s $(190.5 \mathrm{eV})$ and N1s $(398.4 \mathrm{eV})$ spectra for films grown using both processes, in good agreement with previous reports on mono to few-layer BN $[18,19]$.

RHEED was employed to investigate the surface structure and to determine the relationship between film and substrate orientation. Figure 5 shows the RHEED pattern from a $\mathrm{BN}$ film grown with pre-growth nitridation at $1100{ }^{\circ} \mathrm{C}$ and $\mathrm{V} / \mathrm{III}=900$ with the electron beam along the [11-20] (a) and [1-100] (b) of c-plane sapphire, revealing streak patterns characteristic of the $\mathrm{sp}^{2} \mathrm{BN}$ honeycomb surface mesh. These patterns have two distinct separation distances between streaks, which alternate every $30^{\circ}$ of substrate rotation and reappear every $60^{\circ}$. Where 
patterns (a) and (b) of Fig. 5 correspond to the [1-100] and [11-20] crystallographic directions of $\mathrm{sp}^{2} \mathrm{BN}$, consistent with previous reports [20]. Additional information can be collected from the general characteristics of the RHEED patterns. The appearance of only one pattern instead of a superposition of these patterns is consistent with only one lattice orientation reflecting the electron beam at a time. Consequently, the domain sizes are at least the size of the area covered by the incident beam. Moreover, the patterns do not change as the beam is scanned laterally across the surface. From these pattern characteristics we can conclude that the orientation of the BN is fixed with respect to orientation and, therefore, dependent on the sapphire surface orientation. Based on these measurements we have determined the alignment between the BN film and sapphire substrate are [1-100]||[11-20] and [0001]\|[0001].

\section{Discussion}

The effect of surface nitridation on the growth of $\mathrm{sp}^{2} \mathrm{BN}$ is most obvious when comparing the surface morphology of films grown under medium V/III ratios (900 to 1350). Films grown under these conditions without the pre-growth nitridation step form hexagonal and triangular holes, as present in Fig. 2a. From close inspection of these holes, we can see that they tend to be hexagonal or triangular in shape where the edges all align to one of six orientations, forming $60^{\circ}$ or $120^{\circ}$ angles with each other, Fig. 6a. A line scan across one of these holes shows a $1.7 \mathrm{~nm}$ step in agreement with thickness measurements by XRR. The alignment of these holes further indicates the well oriented nature of these films. These types of geometries are reminiscent of the triangular and hexagonal $\mathrm{BN}$ islands grown on metal substrates [20, 22], which has been explained by comparing the different edge terminations [20, 22-25]. The triangular shape $\left(60^{\circ}\right.$ angles $)$ is formed when the edges are all $\mathrm{N}$ or B-terminated, while hexagonal islands $\left(120^{\circ}\right.$ angle) form from edges with alternating B to $\mathrm{N}$-termination where the 
N-terminated edge was found to be the most stable [23]. Based on edge energy calculations [23] and the high V/III used in our process, we would expect a majority of N-terminated edges; however, we observe a large number of holes with $120^{\circ}$ angles between edges indicating a mix of B and N-termination. A schematic of holes with all $\mathrm{N}$-terminated edges and alternating $\mathrm{B}$ and $\mathrm{N}$-terminated edges is presented in Fig. 6c.

AFM images from a film grown for 30 min at these conditions still reveal holes but with much smaller diameters and more triangular type shapes, Fig. 6b. A line scan across one of the larger holes measured a $1.8 \mathrm{~nm}$ step, showing that the thickness is nearly constant with growth time. Increasing growth time does improve lateral coverage noticeably, reducing the average hole size, but did not fill in these holes. A similar scenario was observed in h-BN grown on $\mathrm{Ru}$ where films could not coalesce by simply increasing the growth time [6]. Coalescence was limited by merging of edges with the same termination forming small triangular holes. In holes with adjacent $\mathrm{N}$-terminated edges (those forming $60^{\circ}$ angles), lateral growth results in two edges with the same termination meeting, this stops growth before the films completely coalesce. On the other hand, holes with alternating B and $\mathrm{N}$ edges should merge and form a continuous layer, Fig. 6c. Under these growth conditions films contain holes of both types, so holes will not likely fill in simply by increasing the growth time. These holes, which form due to incomplete coalescence, are eliminated by introduction of the pre-growth nitridation step. Nitriding the surface using the process describe in this paper leads to a higher density of nucleation sites by changing the surface energy of the substrate, which improves the likelihood of coalesces resulting in continuous hole free films.

From the results presented we get a clearer picture of how nitridation effects the growth of few-layer $\mathrm{sp}^{2} \mathrm{BN}$ on sapphire. Like with other highly lattice-mismatched III-nitrides grown 
on sapphire, epitaxial growth occurs by nucleation and coalescence. In the case of $\mathrm{sp}^{2} \mathrm{BN}$ grown at a high V/III ratio, once the substrate surface is completely covered the growth self-terminates. We explain this self-terminating growth by comparing the surface energies of $2 \mathrm{D} B N$ and $3 \mathrm{D}$ sapphire. Since surface energy of a 3D material is much higher than for a 2D surface, the energy barrier for nucleation on a 3D surface is comparatively low; therefore, once the $3 \mathrm{D}$ surface is covered by a 2D material the probability for nucleation decreases leading to termination of growth. Based on growth rates we see that surface nitridation, either by a low-temperature nitridation process or by unintentional nitridation, promotes self-terminating growth. Nitridation also improves surface morphology and coverage, preventing the formation of holes. The $\mathrm{AlO}_{\mathrm{x}} \mathrm{N}_{\mathrm{y}}$ layer, formed using our nitridation process, may serve as a buffer to reduce the lattice mismatch between film and substrate, or to modify the surface energy of the substrate. Similar nitridation layers produced prior to epitaxial growth of $\mathrm{GaN}$ have resulted in increased nucleation density [26,27]. Compared to a clean sapphire surface, $\mathrm{AlO}_{\mathrm{x}} \mathrm{N}_{\mathrm{y}}$ has a higher surface energy [28], which reduces the energy barrier thereby improving nucleation and substrate coverage and promoting self-terminating growth.

\section{Conclusion}

In summary we have studied the effects of sapphire surface nitridation on the epitaxial growth of $\mathrm{sp}^{2} \mathrm{BN}$ by MOCVD. We have developed a controlled process for forming a smooth thin $\mathrm{AlO}_{\mathrm{x}} \mathrm{N}_{\mathrm{y}}$ layer prior to growth of $\mathrm{BN}$ in order to compare the effect on $\mathrm{BN}$ thickness and morphology across a wide range of V/III ratios. Our results show that nitridation improves nucleation, promoting self-terminating growth and improving surface morphology. Without nitridation, films grown at low V/III were covered in hexagonal and triangular holes that could not fill in 
even after 30 min of growth. From RHEED we found the BN to be well aligned to the substrate with orientation relationship of [1-100]॥[11-20].

\section{ACKNOWLEDGMENTS}

This work is funded by Air Force Office of Scientific Research under task number 13RY03COR, Program Manager Dr. Kenneth Goretta. This support is gratefully acknowledged. 


\section{References:}

[1] T. Taniguchi, T. Sato, W. Utsumi, T. Kikegawa, O. Shimomura, Diam. Relat. Matter. 6, 1806 (1997).

[2] K. Michel, B. Verberk, Phys. Status Solidi 246, 2802 (2009).

[3] T. Nakamura, J. Electrochem. Soc. 133, 1120 (1986).

[4] Y. Kobayashi, T. Akasaka, J. Crystal Growth, 3105044 (2008).

[5] M. Chubarov, H. Pedersen, H. Hogberg, J. Jensen, A. Henry, Cryst. Growth Des. 12, (2012) 3215.

[6] W. Auwarter, T. J. Kreutz, T. Greber, J. Osterwalder, Surf. Sci. 429, 229, (1999).

[7 ]M. Corso, W. Auwarter, M. Muntwiler, A. Tamai, T. Greber, J. Osterwalder, Science, 303, 217, (2004).

[8] Q. S. Paduano and M. Snure, Appl. Phys. Exp. 7, 071004, (2014).

[9] M. Chubarov, H. Pedersen, H. Ho“gberg, V. Darakchieva,J. Jensen, P. O. Å. Persson and A. Henry, Phys. Status Solidi RRL, 2011, 5, 397.

[10] K. Uchida, A. Watanabe, F. Yano, M. Kouguchi, T. Tanaka, and S. Minagawa, J. Appl. Phys. 79, 3487 (1996).

[11] T. Hashimoto, Y. Terakoshi, M. Ishida, M. Yuri, O. Imafuji, T. Sugino, A. Yoshikawa, and K. Itoh, J. Cryst. Growth 189-190, 254 (1998).

[12] P. Venne'gue`s and B. Beaumont, Appl. Phys. Lett. 75, 4115 (1999).

[13] M. Seelmann-Eggebert, H. Zimmermann, H. Obloh, R. Niebuhr, and B. Wachtendorf, J. Vac. Sci. Technol. A 16, 2008 (1998).

[14] Y. Cho, Y. Kim, E. R. Weber, S. Ruvimov, and Z. Liliental-Weber, J. Appl. Phys. 85, 7909 (1999).

[15] F. Dwikusuma and T. F. Kuech, J. Appl. Phys. 94, 5656 (2003). 
[16] D. Skuridina, D. V. Dinh, M. Pristovsek, B. Lacroix, M. P. Chauvat, P. Ruterana, M.

Kneissl, P. Voit, Appl. Surf. Sci. 307, 461 (2014).

[17] R. Geick, C. H. Perry, G. Rupprecht, Phys. Rev. 146, 543 (1966).

[18] R. J. Nemanich, S. A. Solin, R. M. Martin, Phys. Rev. B 23, 6348 (1981).

[19] A. Ismach, H. Chou, D. A. Ferrer, Y. Wu, S. McDonnell, H, C. Floresca, A. Covacevich, C.

Pope, R. Piner, M. J. Kim, R. M. Wallace, L. Colombo, and R. S. Ruoff, ACS Nano, 6 (7), 6378 (2012).

[20] R. Y. Tay, M. H. Griep, G. Mallick, S. H. Tsang, R. S. Singh, T. Tumlin, E. H. T. Teo, S. P. Karna, Nano Lett. 14, 839 (2014).

[21] Y. Kobayashi, C. L. Tsai and T. Akasaka, Phys. Status Solidi C, 7, 1906 (2010).

[22] K. K. Kim, A. Hsu, X. Jia, S. M. Kim, Y. Shi, M. Hofmann, D. Nezich, J. F. Rodriguez-

Nieva, M. Dresselhaus, T. Placios, and J. Kong, Nano Lett.12, 161 (2012).

[23] Liu, Y.; Bhowmick, S.; Yakobson, B. I. Nano Lett. 2011, 11 (8), 3113-3116.

[24] W. Auwarter, H. U. Suter, H. Sachdev, and T. Greber, Chem. Mater. 16, 343 (2004).

[25] W. Auwarter, M. Muntwiler, J. Osterwalder, and T. Greber, T. Surf. Sci. 545, L735 (2003).

[26] K. Uchida, A. Watanabe, F. Yano, M. Kouguchi, T. Tanaka and S. Minagawa, J. Appl. Phys.79, 3487 (1996).

[27] M. Ishida, T. Hashimoto, T. Takayama, O. Imafuji, A. Yoshikawa, K. Itoh, Y. Terakoshi, T. Sugino and J. Shirafuji, Mater. Res. Soc. Symp. Proc. 468, 69 (1997).

[28] R. H. French, J. Am. Ceram. Soc. 73, 477 (1990). 


\section{Figure Captions:}

Figure 1: (a) The N1s spectra from the surface of sapphire substrates nitrided from 1 min and 10 min. (b) X-ray reflectance curves from sapphire substrates nitrided for $15 \mathrm{sec}$ to $30 \mathrm{~min}$. (c) AFM images from sapphire surface nitrided for $1 \mathrm{~min}$ and $10 \mathrm{~min}$.

Figure 2: AFM images $(1 \mu \mathrm{m} \times 1 \mu \mathrm{m})$ from BN films grown using V/III ratios from 450 to 2250 (a) without and (b) with the pre-growth nitridation step. (c) Plot of RMS roughness determined by AFM vs. V/III.

Figure 3: (a) X-ray reflectance curves from BN films on sapphire grown using a V/III from 450 to 2700 without the pre-growth nitridation. (b) Thickness vs. V/III for BN films grown on sapphire with and without the pre-growth nitridation process, inset shows thickness vs. growth time for films grown using a V/III ratio of 2250.

Figure 4: (a) Raman spectra from $\mathrm{sp}^{2} \mathrm{BN}$ films on sapphire grown using a high and a low V/III with and without the nitridation process. All spectra are normalized using the sapphire peak at $750 \mathrm{~cm}^{-1}$, and then subtracted sapphire as a background. (b) The B1s and N1s spectra from the surface of BN films on sapphire grown using a V/III of 2250 with and without the nitridation process.

Figure 5: RHEED pattern from BN films on sapphire grown using a V/III of 900 and pre-growth nitridation with the electron beam aligned along the [11-20] (a) and [1-100] of sapphire (b). Lines $a$ and $b$ represent the distance between $B N$ diffraction streaks with $a / b \approx \sqrt{ } 3$.

Figure 6: AFM images from BN film on sapphire grown for $15 \mathrm{~min}$ (a) and $30 \mathrm{~min}(\mathrm{~b})$ using a V/III of 900 without pre-growth nitridation. In sets of (a) and (b) show line-scan indicated by the white line. (c) Schematic depicting hexagonal and triangular holes in the BN lattice. 
Figure 1:

a)
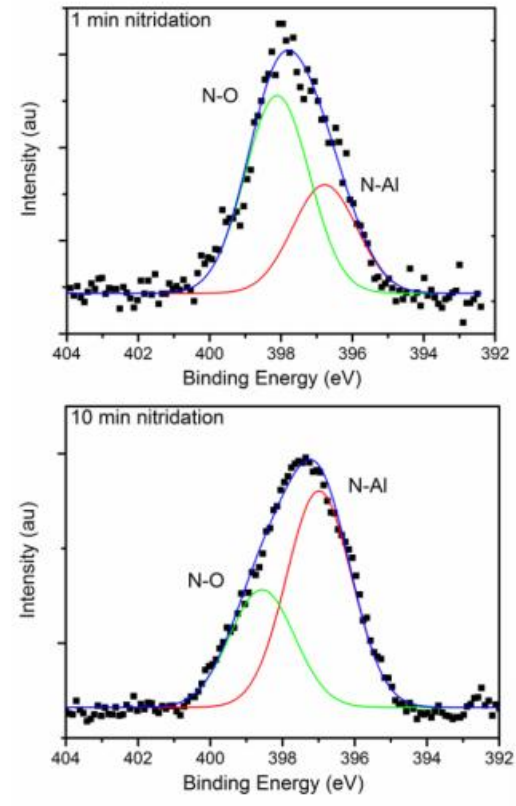

c)

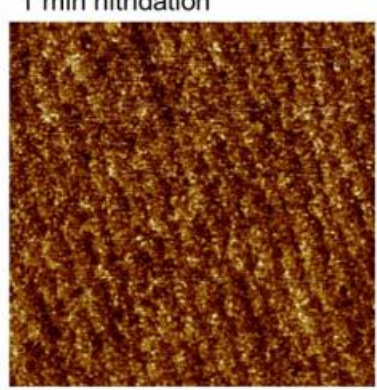

b)

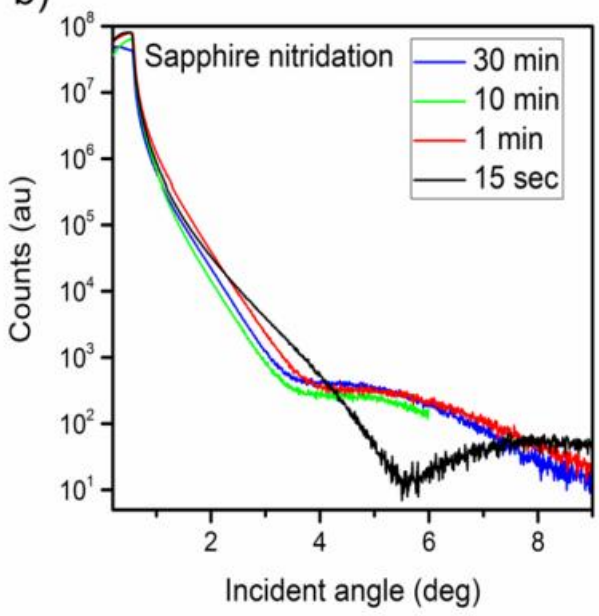

10 min nitridation

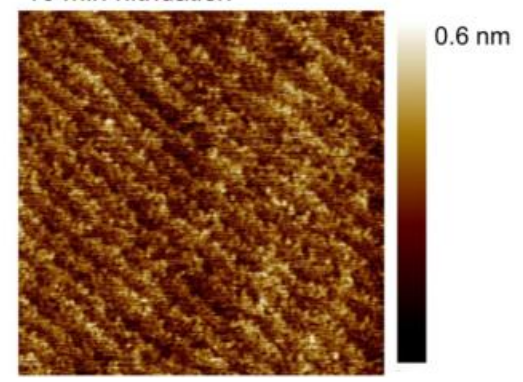


Figure 2:
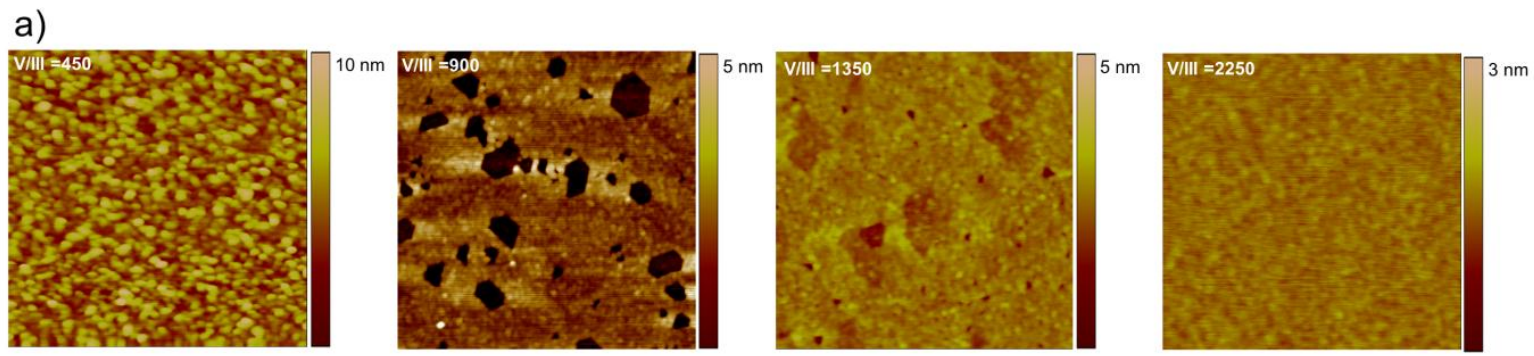

b)
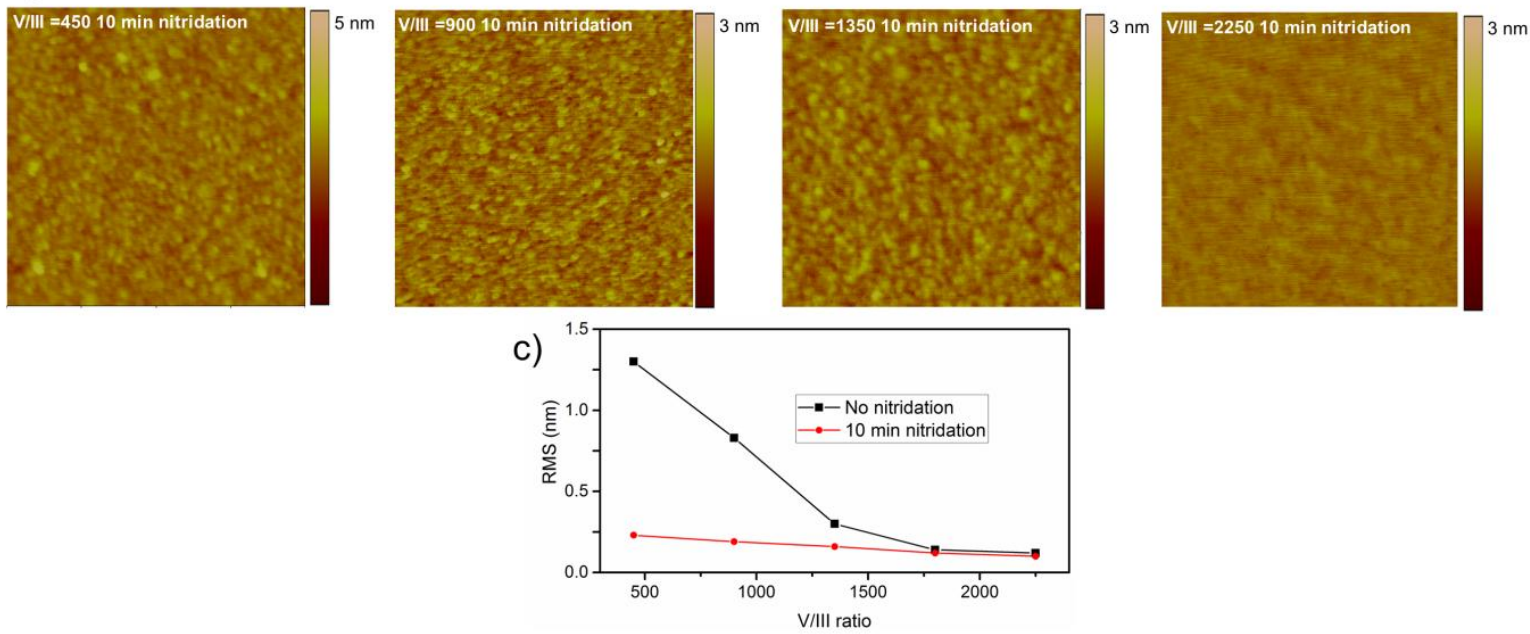
Figure 3:
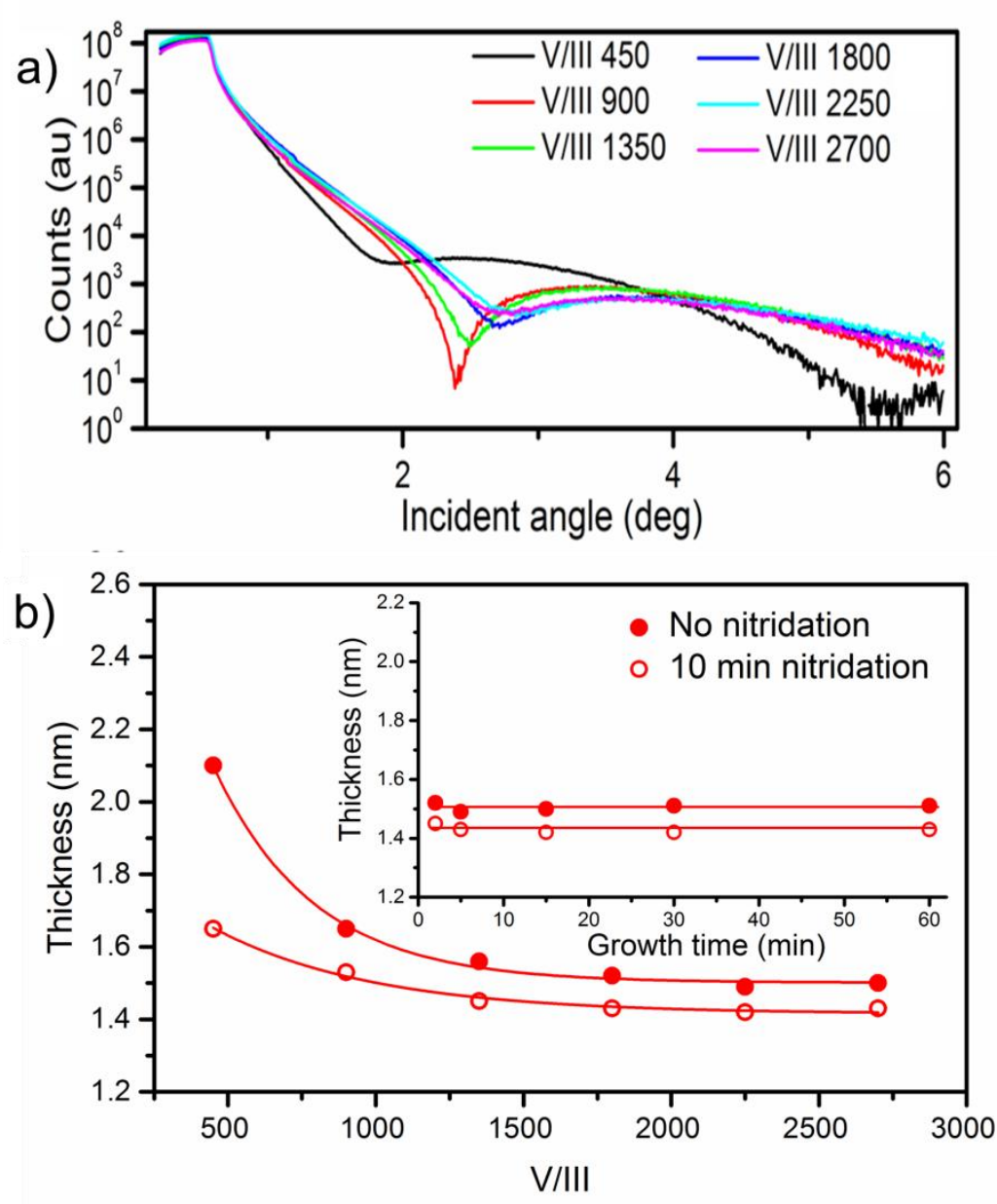
Figure 4:

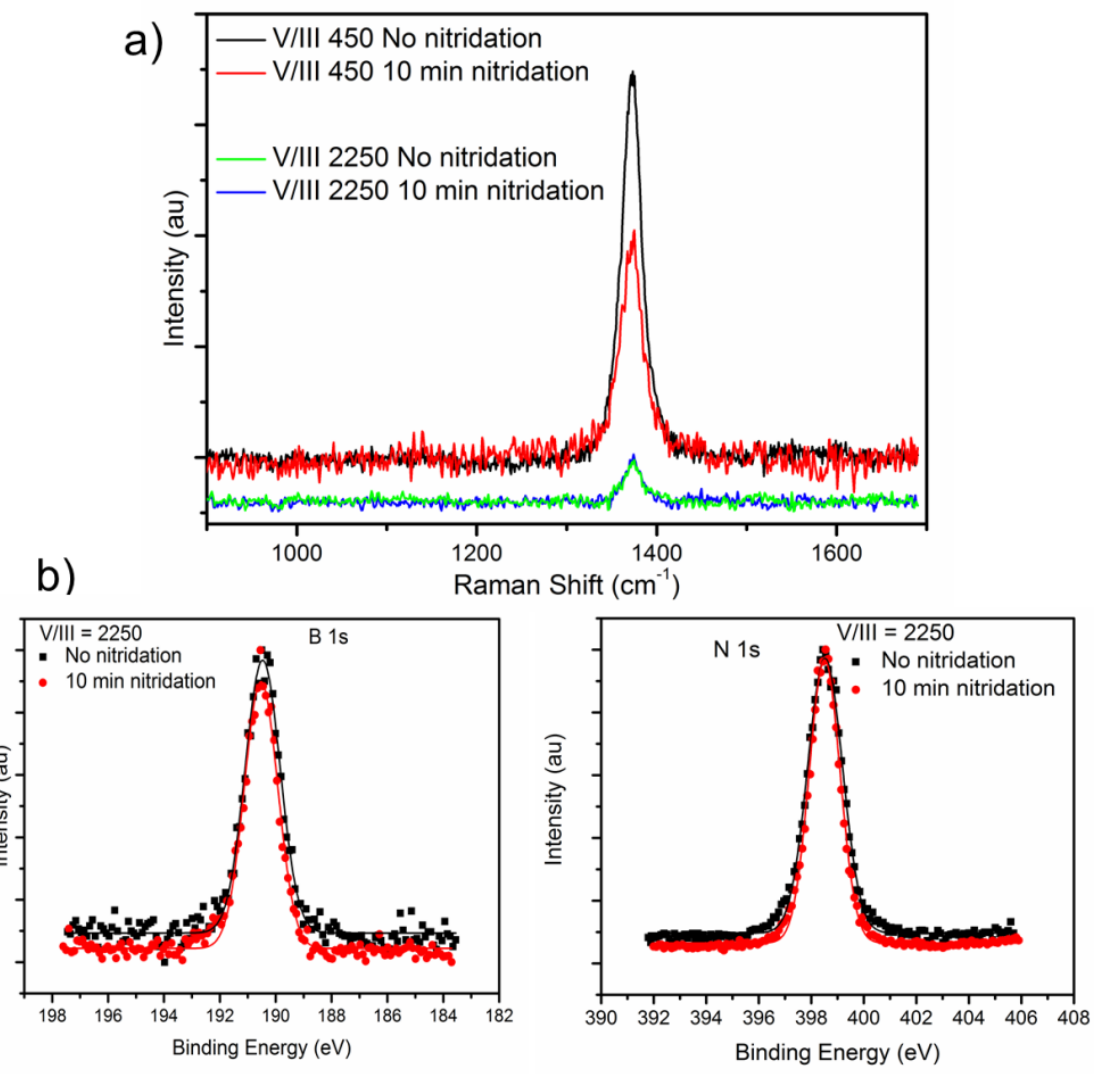


Figure 5:

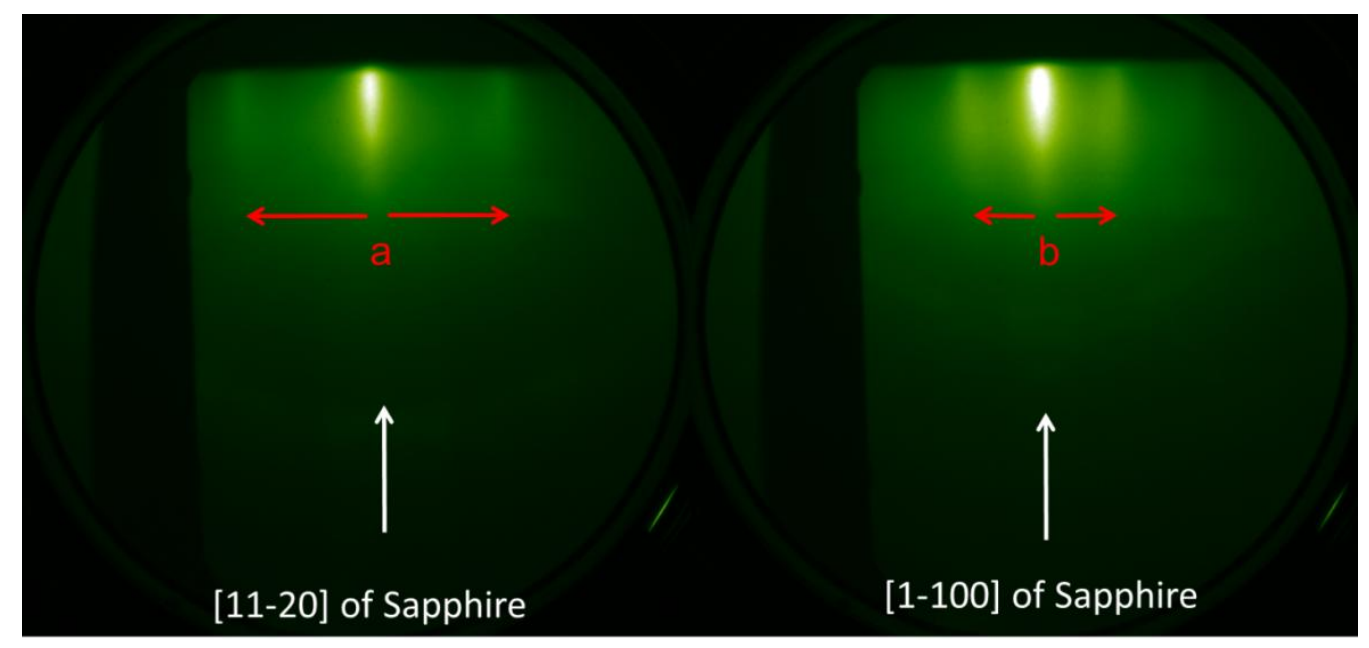


Figure 6:
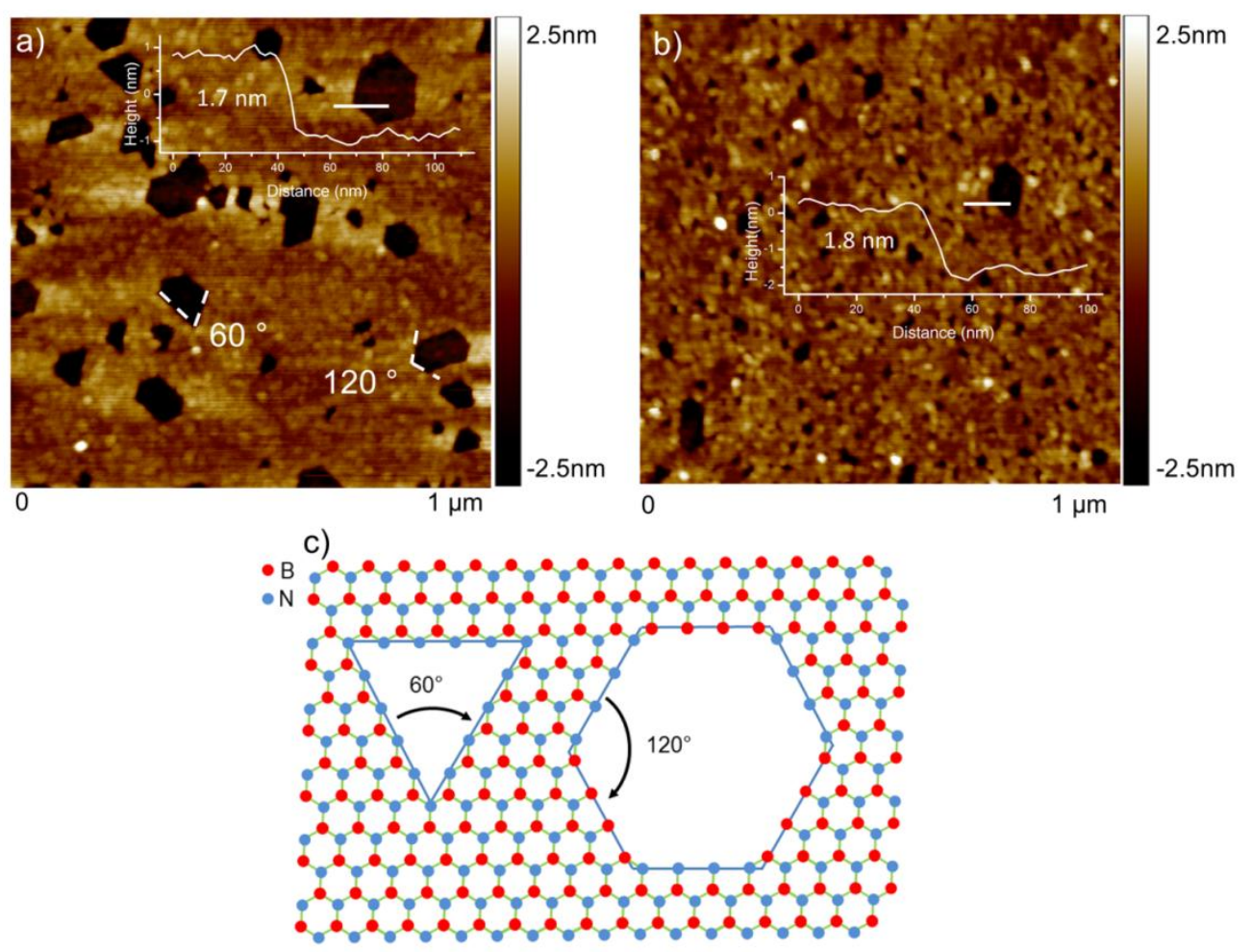